\title{
Observations of playground play during elementary school recess
}

CrossMark

\author{
William V. Massey ${ }^{1 *} \mathbb{D}$, Byungmo Kuㄹ and Megan B. Stellino ${ }^{3}$
}

\begin{abstract}
Objective: The purpose of the current study was to examine reliability and validity evidence for an observational measure of playground play during recess. Observational data of what children played at recess were collected at 236 recess sessions across 26 urban elementary schools. An inductive content analysis of children's type of play and activity engagement during recess was conducted to categorize activities. Inter-rater reliability of observations was assessed at 49 points that spanned 22 unique recess periods at four different schools. Reliability data were collected during the winter and spring seasons. A multivariate analysis of variance was conducted to examine differences in play and activity patterns between genders, and between schools implementing recess interventions (e.g., structured play environment) and schools with no recess intervention.

Results: Results of the content analysis yielded eight playground play and activity categories, all with high levels of inter-rater reliability (ICCS $>.90)$. Significant differences in children's play and activity patterns emerged between genders and across recess intervention conditions. Engagement in 'sports and organized activities' and 'non-engagement in play' contributed most to the separation between boys and girls, while'non-engagement in play' contributed most to the separation between recess intervention and non-intervention schools.
\end{abstract}

Keywords: Play, Recess, Physical activity, School-based health, Reliability, Validity

\section{Introduction}

School-based recess is an opportunity for children to be physically active during the school day [1]. The American Academy of Pediatrics [2] has asserted that in the United States school-based recess not only provides an environment for health enhancing physical activity (PA), but also confers benefits for social development, cognitive functioning, and improved classroom behavior. Yet, results of observational research show that high levels of fighting and conflict can occur during recess, particularly in schools serving high levels of socially disadvantaged students $[3,4]$. There is a need, therefore, to consider the quality of the recess environment, which has recently been operationalized as including the safety and structure

*Correspondence: william.massey@oregonstate.edu

${ }^{1}$ College of Public Health and Human Sciences, School of Biological and Population Health Sciences, Kinesiology Program, Oregon State University, 101 Milam Hall 2520 SW Campus Way, Corvallis, OR 97331, USA Full list of author information is available at the end of the article of the environment, adult engagement and supervision, student social behaviors, and transition periods [5].

Inherent in developing a quality recess environment is understanding children's preferences and opportunities to engage in, and play different games and activities. While previous research has shown differences between boys and girls PA levels at recess [1], and that environmental interventions are effective for increasing PA at recess [6], less attention has been given to children's play preferences that may contribute to both PA promotion, as well as social development during recess. The activities of daily living-playground play (ADL-PP) is an existing measure that was developed for self-reported activities in children older than 7 years of age $[7,8]$. Stellino and Sinclair [9] found that children $(\mathrm{N}=444$; 3rd-5th graders; $49 \%$ female) from a suburban Rocky Mountain region of the USA reported running (79.5\%) and talking with friends $(60.8 \%)$ as the most common activities at recess. However, self-report of activities can be prone to selection or retrieval biases, and Sinclair and Stellino [10] further suggested that this tool may be adaptable

C The Author(s) 2018. This article is distributed under the terms of the Creative Commons Attribution 4.0 International License (http://creativecommons.org/licenses/by/4.0/), which permits unrestricted use, distribution, and reproduction in any medium, provided you give appropriate credit to the original author(s) and the source, provide a link to the Creative Commons license, and indicate if changes were made. The Creative Commons Public Domain Dedication waiver (http://creativecommons.org/ publicdomain/zero/1.0/) applies to the data made available in this article, unless otherwise stated. 
to an observational form of measurement. While previous research has shown that observers can be trained to enhance reliability on the ADL-PP, and that agreement between observer reports and children self-report is generally around 75\% [7], these data were produced in small recess sessions ranging from eight to 29 participants. In considering the observational use of the ADLPP, researchers may be able to examine larger samples of what children do at recess, optimal levels of activity engagement, and better tailor interventions to the needs and general play preferences of children (e.g., more targeted approach to buying equipment and structuring environments). Based on these premises, we used a modified version of the ADL-PP to assess the reliability and validity of an observational measure of children's play patterns.

\section{Main text}

Data in the current study were collected in Milwaukee, WI, USA from 2014 to 2017. A waiver of consent was approved by the institutional review board at Concordia University Wisconsin (ID: 932380-3; 926512-1; 5946225 ) in line with category 1 (standard educational procedures) and category 2 (observations of public behavior) exempted research categories and US Code of Federal Regulations 46.117.

\section{Participants}

Data were collected at 236 different recess sessions across 26 elementary schools. On average, 65 children were present at each recess session, resulting in approximately 16,150 individual observations.

\section{Measures}

The different types of activities children engaged in during recess were measured using an observational form of the ADL-PP [9]. Observations were conducted at 5-min intervals during recess, with frequency counts being collected for all different games, activities, or sedentary behaviors. Throughout data collection, a second coder recorded inter-rater reliability observations at 49 points that spanned 22 unique recess periods at four different schools. To facilitate independent reliability ratings, data assessors were instructed not to discuss frequency counts with other raters, however coordinated their data collection to ensure they conducted their observations at the same time and in the same direction (i.e., counts all took place left to right to control for natural momentary changes in play). Observations for boys and girls were coded separately. Over the course of observations, 80 different activities were recorded including those within the original ADL-PP and those marked in the 'other' category.
Aside from the ADL-PP, observers recorded whether or not an intervention was present on the playground. In total, 107 of the observed recess sessions had a recess intervention present on the playground $(n=82$ recess sessions contracted by Playworks, a national non-profit organization; $n=25$ recess sessions running internal interventions designed by trained physical education teachers).

\section{Data analysis}

Following data collection, an inductive content analysis of activity engagement was conducted by the lead author [11]. Activities that were redundant were combined into a singular category (e.g., straight slide, tube slide, curly slide, were all coded into slide). Next categories of activities were coded into higher order groupings (e.g., slide was coded into jungle gym). These were then sent to the third author for critical peer review with any discrepancies being solved through discussion. Finally, the literature on children's play at recess was reviewed to ensure we included activities known to take place at recess but not observed in this study (e.g., use of cell phones or electronics). The results of this content analysis were the basis for creating an observational protocol for playground play derived from use of the original ADL-PP (Table 1). Next, inter-rater reliability of observational coding for each play domain was tested using a one-way random effects intra-class correlation coefficient (ICC).

To test the construct validity of the tool, differences between gender, and presence of intervention, were examined using a factorial $(2 \times 2)$ multivariate analysis of variance (MANOVA) test in SAS 9.4 (@SAS Institute, Inc., North Carolina, USA). Based on previous research, it was hypothesized that different patterns would emerge between boys and girls, and between children at intervention schools and non-intervention schools $[1,6]$. Canonical coefficients were also computed to determine the relative contribution (i.e., largest, smallest) of each dependent variable to the identified discriminant function. An alpha of .05 was used to determine statistical significance for all omnibus tests, and reported effect sizes $\left(\eta^{2}\right)$ were interpreted as small $\left(\eta^{2}=.01\right)$, medium $\left(\eta^{2}=.06\right)$, or large $\left(\eta^{2}=.14\right)$. Post-hoc univariate tests were then conducted. The relative benefit increase of the presence of an intervention for boys and girls separately as it relates to engagement during recess (Engagement Rate at Intervention Schools-Engagement Rate at NonIntervention Schools/Engagement Rate at Non-Intervention Schools) was also examined.

Statistical assumptions were checked prior to conducting the omnibus tests. Three categories (anti-social behaviors; rough and tumble play; nature) violated normality assumptions, which was likely based on low 
Table 1 "Observation of Playground Play" (OPP) tool for use in coding children's behavior

\begin{tabular}{|c|c|}
\hline Playing on equipment (frea & \\
\hline Jungle gym & Boys \\
\hline Swings & Boys \\
\hline Rock climbing & Boys \\
\hline Other & Boys \\
\hline Organized sports and activi & \\
\hline Basketball & Boys \\
\hline Football & Boys \\
\hline Kickball & Boys \\
\hline Soccer & Boys \\
\hline Dance & Boys \\
\hline Other & Boys \\
\hline Active and chase games (fre & \\
\hline Tag/chasing & Boys \\
\hline Racing & Boys \\
\hline Running & Boys \\
\hline Other & Boys \\
\hline Traditional playground gam & \\
\hline Four square & Boys \\
\hline Hula-hoops & Boys \\
\hline Jump rope & Boys \\
\hline Tetherball & Boys \\
\hline Hopscotch & Boys \\
\hline Unfixed equipment & Boys \\
\hline Other & Boys \\
\hline Nature (frequency count) & \\
\hline Imaginative play & Boys \\
\hline Digging & Boys \\
\hline Climbing hills & Boys \\
\hline Playing with bugs & Boys \\
\hline Other & Boys \\
\hline Rough and tumble play (fre & \\
\hline Wrestle & Boys \\
\hline Play fight & Boys \\
\hline Fence climbing & Boys \\
\hline Other & Boys \\
\hline Anti-social behavior (freque & \\
\hline Fighting & Boys \\
\hline Pushing/shoving/hitting & Boys \\
\hline Timeout/wall & Boys \\
\hline Yelling & Boys \\
\hline Other & Boys \\
\hline Non-engagement in active & \\
\hline Standing around & Boys \\
\hline Talking with friends & Boys \\
\hline Electronics & Boys \\
\hline Sit-down game & Boys \\
\hline Other & Boys \\
\hline
\end{tabular}

prevalence rates. Nonetheless, we viewed these categories as having practical significance in considering children's recess play activities. Given previous reports that ANOVA based analyses are robust to violations of normality [12,13] in conjunction with issues surrounding $\log$ transformations of frequency data $[14,15]$, the decision was made to not transform the data to meet normality assumptions, as this could compromise meaningful interpretation of the data.

\section{Results}

Content analyses of the activities children engaged in during recess yielded eight categories of play: (1) playing on equipment, (2) organized sports and activities, (3) active and chasing games, (4) traditional playground games, (5) nature, (6), rough and tumble play, (7) antisocial behavior, and (8) non-engaged in play. Table 1 depicts a new observational form derived from the ADLPP, the Observation of Playground Play (OPP). Inter-rater reliability coefficients for each category are reported in Table 2.

Descriptive statistics for children's playground play engagement patterns were calculated and are reported in Table 2. The factorial MANOVA test result revealed a non-significant interaction effect, $F(8,461)=1.85$, $\left.p=.07, \Lambda=.97, \eta^{2}=.03\right)$. A significant main effect of gender was identified, $\mathrm{F}(8,461)=41.32, \mathrm{p}<.001, \Lambda=.58$, $\eta^{2}=.42$. The standardized canonical coefficients reported in Table 3 revealed that in the context of all eight play categories, organized sports and activities, and nonengagement contributed most to the separation between males and females. Post hoc tests revealed that boys engaged in significantly lower levels of non-engagement $\mathrm{t}(470)=13.198, \mathrm{p}<.001$, traditional playground games $\mathrm{t}(470)=2.426, \mathrm{p}=.016$, active and chase games $\mathrm{t}(470)=2.559, \mathrm{p}=.011$, and higher levels of organized sports and activities $\mathrm{t}(470)=-17.898, \mathrm{p}<.001$, when compared to girls. A significant main effect for intervention was also identified, $F(8,461)=5.26, \mathrm{p}<.001$, $\Lambda=.916, \eta^{2}=.08$. The standardized canonical coefficients revealed that non-engagement contributed most to the separation between schools with and without an intervention. Post hoc tests revealed that children at intervention schools engaged in higher levels of playing on equipment $\mathrm{t}(470)=-3.789, \mathrm{p}<.001$ and traditional playground games $\mathrm{t}(470)=-6.991, \mathrm{p}<.001$, and lover levels of non-engagement $\mathrm{t}(470)=8.555, \mathrm{p}<.001$ and anti-social behavior $\mathrm{t}(421.53)=2.152, \mathrm{p}=.032$. Finally, at schools with an intervention present, there was a relative benefit increase of $45.8 \%$ to reduce non-engagement for boys and a relative benefit increase of $42 \%$ to reduce nonengagement for girls. 
Table 2 Descriptive statistics and reliability indices

\begin{tabular}{|c|c|c|c|c|c|c|}
\hline OPP play domain & Total & Boys & Girls & Intervention & Non-intervention & Inter-rater reliability \\
\hline Playing on equipment & $11.38 \%$ & $10.51 \%$ & $12.25 \%$ & $14.15 \%^{++}$ & $9.09 \%$ & $I C C=.986$ \\
\hline Organized sports and activities & $26.54 \%$ & $41.60 \%+$ & $11.48 \%$ & $26.61 \%$ & $26.48 \%$ & $\operatorname{ICC}=.965$ \\
\hline Active and chase games & $14.51 \%$ & $12.91 \%^{+}$ & $16.11 \%$ & $15.14 \%$ & $13.98 \%$ & $\operatorname{ICC}=.969$ \\
\hline Traditional playground games & $15.74 \%$ & $14.10 \%^{+}$ & $17.39 \%$ & $20.72 \%^{++}$ & $11.61 \%$ & $I C C=.939$ \\
\hline Nature & $1.38 \%$ & $1.14 \%$ & $1.63 \%$ & $1.70 \%$ & $1.12 \%$ & $\operatorname{ICC}=.999$ \\
\hline Rough and tumble play & $1.27 \%$ & $1.00 \%$ & $1.61 \%$ & $1.07 \%$ & $1.43 \%$ & $I C C=.972$ \\
\hline Anti-social behavior & $1.72 \%$ & $1.78 \%$ & $1.67 \%$ & $1.17 \%^{++}$ & $2.18 \%$ & $I C C=.999$ \\
\hline Non-engagement in active play & $27.49 \%$ & $17.06 \%^{+}$ & $37.92 \%$ & $19.40 \%^{++}$ & $34.20 \%$ & $I C C=.976$ \\
\hline
\end{tabular}

+ Significant difference between boys and girls

++ Significant differences between intervention and non-intervention

Table 3 Canonical coefficients associated with gender and intervention contrasts

\begin{tabular}{lcc}
\hline OPP play domain & Gender standardized canonical coefficient & Intervention standardized canonical coefficient \\
\hline Playing on equipment & -.126 & .709 \\
Organized sports and activities & .626 & 1.264 \\
Active and chase games & -.094 & .562 \\
Traditional playground games & -.294 & .536 \\
Nature & -.125 & .440 \\
Rough and tumble play & -.138 & .591 \\
Anti-social behavior & .200 & .274 \\
Non-engagement in active play & -.903 & 2.076 \\
\hline
\end{tabular}

\section{Discussion}

The current study extends the school-based (PA) measurement literature. Evidence is provided for an observational measure of children's playground play across eight activity categories [16]. The Observations of Playground Play (OPP) measure provides a practical tool for researchers, and practitioners, to better understand playground activity and play patterns in an outdoor environment. In examining playground play patterns, the data in the current study help to elucidate how differential play patterns might explain discrepancies in recess PA, and how interventions might play a role in reducing this gap. Specifically, while PA has been shown to increase physical health, as well as cognitive performance [17], data directly linking recess PA to social, cognitive, or academic outcomes remains elusive [18]. Thus, the type of activity, and the social-emotional implications of engagement (e.g., fighting versus participation in a team sport) are likely to moderate various outcomes, and the use of the $O P P$ can help researchers and practitioners better understand how play preferences shape the recess environment.

In terms of validity, the activity patterns in the current study are consistent with previous studies, indicating that gender significantly impacts children's patterns of activity engagement during recess $[1,19,20]$. In the current study, boys were significantly more involved in organized sports and activities (e.g., soccer and football) and participated less in non-engagement activity (e.g., talking with friends and sit-down games) compared to girls. This finding supports results from previous research [9], that indicated boys participated in more sport-related activities and girls were more engaged in non-active play (e.g., talking), or use of equipment (e.g., swinging). The difference in activity between boys and girls found in the present study is also consistent with other previous findings that examined the meaning of recess for boys and girls. Notably, a study found that boys consider recess as a time for participating in sports-related activity but girls consider it as a time for participating in sedentary-related social activity [21].

Examining differences between intervention and nonintervention schools also supported the construct validity of the OPP. Compared to children at recesses with no intervention present, children in recesses with an intervention engaged in playing on equipment significantly more, and showed patterns of non-engagement in active play and anti-social behaviors significantly less. This is in line with previous research that showed recess interventions including, but not limited to adding equipment, 
play markings, zones, and teacher engagement, are effective for promoting PA [16]. Furthermore, and aligned with previous research, the standardized canonical coefficients reported in the current study revealed that non-engagement in active play contributed most to the separation between schools with, and without, an intervention.

\section{Limitations}

An important limitation of the current study is that schools represented one geographic region of the United States, and the patterns of recess play and activity observed in the current study might not be representative of a broader and/or more national, or international, population. Additional limitations include a lack of concurrent validity of recess play and PA patterns and a lack of detail specifying how the environment shaped various those patterns. Finally, due to the time sampling nature of frequency counts as a mode of observation, it is likely that more sporadic behaviors (e.g., pushing and shoving) are under-represented.

\section{Authors' contributions}

WVM was involved in study conceptualization, oversaw all data collection, and led data analysis and interpretation. BMK was involved with data analysis and interpretation and writing of the manuscript. MBS lead study conceptualization, was involved in interpretation of data analysis, and contributed to the writing of the paper. All authors read and approved the final manuscript.

\section{Author details}

${ }^{1}$ College of Public Health and Human Sciences, School of Biological and Population Health Sciences, Kinesiology Program, Oregon State University, 101 Milam Hall 2520 SW Campus Way, Corvallis, OR 97331, USA. ${ }^{2}$ College of Public Health and Human Sciences, School of Biological and Population Health Sciences, Kinesiology Program, Oregon State University, 008 Women's Building, Corvallis, OR 97331, USA. ${ }^{3}$ School of Sport and Exercise Science, University of Northern Colorado, Greeley, CO 80639, USA.

\section{Acknowledgements}

We would like to extend our appreciation to all of the school districts that have partnered with us in this research. We would also like to thank Rachel Rodia, Kelsey Dykema, Sheri Matitz, Alex Ross, Maggie Fraser, Shannon Magnuson, Alexa Wistenberg, Tyger Gruber and Stephanie Le for their assistance on this project.

\section{Competing interests}

The authors declare that they have no competing interests.

\section{Availability of data and materials}

The datasets used and/or analyzed during the current study are available from the corresponding author on reasonable request.

\section{Consent for publication}

Not applicable.

\section{Ethics approval and consent to participate}

Ethics approval was provided by the institutional review board at Concordia University Wisconsin (ID: 932380-3; 926512-1; 594622-5). The need for consent was waived as no individual level, or identifiable data were collected. WVM was a faculty member at Concordia University Wisconsin from 2014 to 2017.

\section{Funding}

This work was funded in part by grants from the Burke Foundation and Playworks Education Energized.

\section{Publisher's Note}

Springer Nature remains neutral with regard to jurisdictional claims in published maps and institutional affiliations.

Received: 3 July 2018 Accepted: 17 October 2018

Published online: 23 October 2018

\section{References}

1. Ridgers ND, Salmon J, Parrish A-M, Stanley RM, Okely AD. Physical activity during school recess. Am J Prev Med. 2012;43(3):320-8.

2. Council on School Health. The crucial role of recess in school. Pediatrics. 2013;131(1):183-8.

3. Massey WV, Stellino MB, Holliday M, Godbersen T, Rodia R, Kucher G, et al. The impact of a multi-component physical activity programme in lowincome elementary schools. Health Educ J. 2017;76(5):517-30.

4. Ridgers ND, Fairclough SJ, Stratton G. Variables associated with children's physical activity levels during recess: the A-CLASS project. Int I Behav Nutr Phys Act. 2010;7(1):74.

5. Massey WV, Stellino MB, Mullen SP, Claassen J, Wilkison M. Development of the great recess framework - observational tool to measure contextual and behavioral components of elementary school recess. BMC Public Health. 2018. https://doi.org/10.1186/s12889-018-5295-y.

6. Ickes MJ, Erwin H, Beighle A. Systematic review of recess interventions to increase physical activity. J Phys Act Health. 2013;10(6):910-26.

7. Watkinson EJ, Dunn JC, Cavaliere N, Calzonetti K, Wilhelm L, Dwyer S. Engagement in playground activities as a criterion for diagnosing developmental coordination disorder. Adap Phys Act Q. 2001;18(1):18-34.

8. Watkinson E, Muloin S. An instrument for the assessment and prescription of skills of mentally handicapped children. Ottawas: Report to The Canadian Firness and Lifestyle Rexearch Institute; 1989.

9. Stellino MB, Sinclair C. Examination of children's recess physical activity patterns using the activities for daily living-playground participation (ADL-PP) instrument. J Teach Phys Educ. 2014;33(2):282-96.

10. Sinclair C, Babkes Stellino M. Pictorial playground-based physical activity assessment instrument: uses and applications of the ADL-PP. J Phys Educ Recreat Dance. 2017;88(3):16-21.

11. Tashakkori C, Tashakkori A. Overview of contemporary issues in mixed methods research. Handbook of mixed method in social and behavioral research. 2010. p. 1-41.

12. Lix LM, Keselman JC, Keselman HJ. Consequences of assumption violations revisited: a quantitative review of alternatives to the one-way analysis of variance $F$ test. Rev Educ Res. 1996;66(4):579-619.

13. Harwell MR, Rubinstein EN, Hayes WS, Olds CC. Summarizing monte carlo results in methodological research: the one- and two-factor fixed effects ANOVA cases. J Educ Stat. 1992;17(4):315-39.

14. O'Hara RB, Kotze DJ. Do not log-transform count data: do not log-transform count data. Methods Ecol Evol. 2010;1(2):118-22.

15. Lo S, Andrews $\mathrm{S}$. To transform or not to transform: using generalized linear mixed models to analyse reaction time data. Front Psychol. 2015. https://doi.org/10.3389/fpsyg.2015.01171/abstract.

16. Cicchetti DV. Guidelines, criteria, and rules of thumb for evaluating normed and standardized assessment instruments in psychology. Psychol Assess. 1994;6(4):284-90.

17. Hillman CH, Pontifex MB, Castelli DM, Khan NA, Raine LB, Scudder MR et al. Effects of the FITKids randomized controlled trial on executive control and brain function. Pediatrics. 2014;134(4):e1063-71.

18. Bundy A, Engelen L, Wyver S, Tranter P, Ragen J, Bauman A, et al. Sydney playground project: a cluster-randomized trial to increase physical activity, play, and social skills. J Sch Health. 2017;87(10):751-9.

19. Saint-Maurice PF, Welk GJ, Silva P, Siahpush M, Huberty J. Assessing children's physical activity behaviors at recess: a multi-method approach. Pediatr Exerc Sci. 2011;23(4):585-99.

20. Woods AM, Graber K, Daum D. Children's recess physical activity: movement patterns and preferences. J Teach Phys Educ. 2012;31(2):146-62.

21. Blatchford $P$, Baines E, Pellegrini A. The social context of school playground games: sex and ethnic differences, and changes over time after entry to junior school. Br J Dev Psychol. 2003;21(4):481-505. 